\title{
Vers une analyse factorielle de l'alternance indicatif/subjonctif
}

\author{
Pascal Amsili et Floriane Guida \\ Laboratoire de Linguistique Formelle \\ Université Paris Diderot \\ amsili@linguist.univ-paris-diderot.fr
}

\section{Introduction}

«La concurrence du subjonctif et de l'indicatif demeure envers et contre tout l'un des trois ou quatre filons inépuisables de la linguistique française », si l'on en croit Wilmet (1997). Nous pensons pourtant qu'il est utile aujourd' hui de revenir sur cette question (nous parlerons d'alternance) dans la mesure où les méthodes expérimentales et quantitatives récemment arrivées dans le champ de la linguistique permettent de revisiter ce problème d'un façon fructueuse.

Précisons tout d'abord ce que nous entendons par alternance indicatif/subjonctif : il s'agit de contextes linguistiques dans lesquels les locuteurs semblent placés devant un choix entre le mode subjonctif et le mode indicatif. Nous ne considérons donc pas les nombreux contextes où en français contemporain le mode (subjonctif ou indicatif) est fixé sans ambiguïté par les constructions. Pour n'évoquer que des situations de subordination, ${ }^{1}$ on reconnaît aisément dans la grammaire trois types de situation :

- L'indicatif est le seul mode possible (1a), (2a);

- Le subjonctif est le seul mode possible (1b), (2b);

- Le subjonctif et l'indicatif sont tous les deux possibles dans un contexte identique : ce sont les cas d'alternance (1c), (2c).

(1) a. Max est certain que Paul $\left\{\right.$ vient $_{\mathrm{IND}} / *^{*}$ vienne $\left._{\mathrm{SBJ}}\right\}$.

b. Tout le monde souhaite que Paul $\left\{*_{\text {vient }}{ }_{\mathrm{IND}} /\right.$ vienne $\left._{\mathrm{SBJ}}\right\}$.

c. Que Paul $\left\{\right.$ vient $_{\mathrm{IND}} /$ vienne $\left._{\mathrm{SBJ}}\right\}$, personne n'en doute.

(2) a. Claude croit que Pierre $\left\{\mathrm{est}_{\mathrm{IND}} / \mathrm{soit}_{\mathrm{SBJ}}\right\}$ parti.

b. Claude veut que Pierre $\left\{*\right.$ part $_{\mathrm{IND}} /$ parte $\left._{\mathrm{SBJ}}\right\}$.

c. Claude ne croit pas que Pierre $\left\{\mathrm{est}_{\mathrm{IND}} / \mathrm{soit}_{\mathrm{SBJ}}\right\}$ parti.

Nous nous intéressons dans cet article à un de ces cas d'alternance, illustré en (2), qui implique les complétives de certains prédicats épistémiques sous la négation.

Les situations d'alternance provoquent des discussions de deux ordre parmi les linguistes : d'une part des discussions à propos de la réalité de l'alternance : peut-être sous l'influence d'une vision prescriptive, ou peut-être sous l'influence des cas particuliers étudiés par les uns ou les autres, il y a fréquemment débat sur les données elles-mêmes (sur le fait que les formes en alternance sont ou non également grammaticales, ou sur les effets sémantiques ou leur absence, etc.). Il y a bien sûr aussi discussion sur l'explication du phénomène : cela dépend évidemment de la première discussion, mais dans beaucoup de cas il s'agit de chercher une explication unifiée, catégorique, du phénomène. 
Nous voulons dans cet article poser les bases d'une approche alternative : pour cela, il faut revenir d'abord sur les faits linguistiques. C'est ce qui motive l'expérience de production induite qui est présentée dans cet article. Les contraintes de la démarche expérimentale conduisent à nous focaliser sur un seul aspect de l'alternance, mais nous espérons pouvoir poursuivre cette démarche de mise au jour de données linguistiques robustes, indispensables pour proposer une analyse du phénomène. Par ailleurs, nous pensons qu'il faut aussi prendre au sérieux la dimension non catégorique et multi-factorielle du phénomène, et nous proposons une série de facteurs, sans prétendre à l'exhaustivité, qui fondent le plaidoyer que constitue cet article en faveur d'une analyse statistique du phénomène de l'alternance.

Nous présentons d'abord l'alternance en jeu (section 2), en précisant quelques-unes des propriétés linguistiques pertinentes d'après la littérature, et en résumant les propositions d'explications qui ont pu être faites ; dans une deuxième partie nous décrivons une étude expérimentale dont nous commentons les résultats (section 3); et nous terminons en présentant une liste de facteurs dont nous pensons qu'ils peuvent être pertinents (section 4).

\section{Description de l'alternance}

Nous donnons d'abord une description de l'alternance qui nous intéresse, en élicitant les facteurs linguistiques pertinents, avant de résumer — succinctement, faute de place — les propositions théoriques qui se sont présentées.

\subsection{Phénomène}

L'alternance qui nous intéresse ici met en jeu des prédicats épistémiques exprimant une croyance positive, placés dans un contexte qui suspend l'opérativité de cette croyance; c'est typiquement le cas de la phrase négative (3b) ou de la phrase interrogative (3c). Dès que la positivité de la croyance est remise en cause, le subjonctif devient possible, alors que l'indicatif est seul possible en contexte canonique déclaratif positif (3a). Nous appellerons CAN (canonique) les contextes comme (3a), et NON-AFF (non affirmatif) les contextes comme (3b) ou (3c).

(3) a. Marie croit que sa camarade $\left\{\mathrm{est}_{\mathrm{IND}} /\right.$ soit $\left._{\mathrm{SBJ}}\right\}$ arrivée

b. Marie ne croit pas que sa camarade $\left\{\mathrm{est}_{\mathrm{IND}} /\right.$ soit $\left._{\mathrm{SBJ}}\right\}$ arrivée.

c. Marie croit-elle que sa camarade $\left\{\right.$ est $_{\mathrm{IND}} /$ soit $\left._{\mathrm{SBJ}}\right\}$ arrivée ?

\subsubsection{Prédicats}

Les prédicats concernés sont des prédicats épistémiques, comme les verbes penser, croire, trouver, avoir l'impression, estimer, considérer..., les adjectifs sûr, certain..., qui peuvent entrer dans des constructions attributives personnelles (Jean est sûr que...) ou impersonnelles (il n'est pas certain que...). À cette classe de prédicats que nous nommerons prédicats épistémiques modaux, faute d'une meilleure terminologie (on les appelle aussi prédicats d'opinion), on peut ajouter les verbes implicatifs simples (ainsi appelés par Mørdrup (1975)) comme démontrer, prouver, faire que..., qui donnent lieu à une alternance du même type (4). Les prédicats épistémiques factifs comme savoir, les semi-factifs comme découvrir, réaliser, qui demandent l'indicatif, ne donnent pas lieu à l'alternance (5). Il en est de même des prédicats factifs émotifs, comme regretter, apprécier, déplorer, qui demandent (toujours) le subjonctif (6).

(4) a. Paul a prouvé que la terre $\left\{\operatorname{est}_{\mathrm{IND}} /\right.$ soit $\left._{\mathrm{SBJ}}\right\}$ ronde.

b. Personne n'a prouvé que la terre $\left\{\operatorname{est}_{\mathrm{IND}} / \mathrm{soit}_{\mathrm{SBJ}}\right\}$ ronde. 
c. Ces données ont fait que la décision $\left\{\mathrm{est}_{\mathrm{IND}} / *^{*} \mathrm{soit}_{\mathrm{SBJ}}\right\}$ prise.

d. Ces données n'ont pas fait que la décision $\left\{\mathrm{est}_{\mathrm{IND}} / \mathrm{soit}_{\mathrm{SBJ}}\right\}$ prise.

(5) a. Jean ne sait pas que Marie $\left\{\right.$ connaît $_{\mathrm{IND}} / *$ connaisse $\left._{\mathrm{SBJ}}\right\}$ le Morbihan.

b. Personne n'a réalisé que Max $\left\{\mathrm{est}_{\mathrm{IND}} / * \mathrm{soit}_{\mathrm{SBJ}}\right\}$ malade.

(6) a. Tout le monde regrette que $\operatorname{Max}\left\{* \operatorname{est}_{\mathrm{IND}} / \mathrm{soit}_{\mathrm{SBJ}}\right\}$ malade.

b. Personne ne regrette que $\operatorname{Max}\left\{*^{e} \mathrm{est}_{\mathrm{IND}} / \mathrm{soit}_{\mathrm{SBJ}}\right\}$ malade.

Il n'est pas évident que les prédicats permettant l'alternance forment une classe naturelle homogène, d'autant que d'autres cas isolés ont été relevés dans la littérature. Il faut noter aussi que les prédicats qui acceptent une alternance de mode dans les complétives sous une matrice négative ne sont pas exactement les mêmes que ceux qui acceptent une alternance dans les complétives disloquées (illustrée en (1c)). Nous nous limitons aux prédicats épistémiques modaux dans la suite de ce texte.

Ces prédicats sont majoritairement non factifs, et le contraste est net avec les factifs et semi-factifs illustrés à l'instant, qui ne donnent pas lieu à une alternance, mais il existe pourtant, dans la liste des verbes factifs dits $\alpha$ de (Mørdrup, 1975) des verbes comme se souvenir, se rappeler qui donnent naissance à l'alternance de mode sous la négation (7). Ce fait nous mène à conclure que la (non) factivité n'est pas un critère pertinent pour caractériser les prédicats d'alternance.

(7) Alix ne se souvient pas que Luc $\left\{\right.$ fait $_{\mathrm{IND}} /$ fasse $\left._{\mathrm{SBJ}}\right\}$ partie du groupe.

\subsubsection{Temps verbaux}

L'indicatif présent peut alterner avec le subjonctif présent, comme en (6). Le subjonctif présent peut également alterner avec le futur de l'indicatif, comme en (8). Enfin, le passé composé de l'indicatif peut alterner avec le passé du subjonctif, comme en (9).

(8) Jean n'est pas certain/sûr que Claude $\left\{\right.$ viendra $_{\text {IND-FUT }} /$ vienne $\left._{\text {SBJ-PRES }}\right\}$ le voir.

(9) Jean ne croit pas que Claude $\left\{\mathrm{a}_{\mathrm{IND}-\mathrm{PRES}} / \mathrm{ait}_{\mathrm{SBJ}-\mathrm{PAS}}\right\}$ fait ça.

Lorsque le subjonctif présent alterne avec le futur (périphrastique ou synthétique) de l'indicatif, il a une interprétation future. L'interprétation future se détermine en contexte. Ainsi, dans (10), le syntagme « un jour » conditionne une interprétation future, et donc l'analyse du verbe comme un subjonctif présent.

Je ne pense pas que tu la revoies ${ }_{\mathrm{SBJ} \text {-PRES }}$ un jour.

(J.-C. Izzo, Chourmo, 1996 ; Frantext)

Il n'y a pas vraiment d'alternance, a priori, pour les autres temps de l'indicatif, comme l'imparfait, puisqu'ils n'ont pas de «correspondant» dans le mode subjonctif. En effet, si le subjonctif imparfait était encore courant au XIX ${ }^{\mathrm{e}}$ siècle, ce n'est plus guère le cas aujourd'hui. On le rencontre encore à l'écrit, dans les textes littéraires par exemple (11a), mais il est extrêmement rare de le rencontrer à l'oral; son emploi est alors la marque d'un registre très, voire extrêmement, soutenu.

(11) a. Je n'étais pas sûr que la Photographie existât $t_{\mathrm{SBJ}-\mathrm{IMP}}$, qu'elle disposât ${ }_{\mathrm{SBJ}-\mathrm{IMP}}$ d'un 'génie' propre. (R. Barthes, La chambre claire. Note sur la photographie, 1980 ; Frantext)

b. Je n'étais pas sûr que la Photographie existait ${ }_{\text {IND-IMP }}$, qu'elle disposait ${ }_{\text {IND-IMP }}$ d'un génie propre.

c. Je n'étais pas sûr que la Photographie existe ${ }_{\mathrm{SBJ} \text {-PRES }}$, qu'elle dispose $\mathrm{SBJ}_{\mathrm{SB} \text {-PRES }}$ d'un génie propre. 
Aujourd'hui, l'indicatif imparfait est donc employé facilement dans les contextes NON-AFF (11b). Cependant, dans certains cas, le subjonctif présent peut prendre le relais de l'imparfait (11c). Le subjonctif présent, qui n'inscrit pas le procès dans une époque particulière, semble apte à exprimer toutes les époques. L'alternance est donc plus difficile à décrire qu'il n'y paraît, puisque le subjonctif présent peut alterner avec différents temps de l'indicatif.

\subsubsection{Contextes}

Le contexte le plus favorable au subjonctif est, semble-t-il, le contexte négatif. On mentionne aussi souvent le contexte interrogatif, dans lequel l'emploi des modes serait très dépendant de la forme de l'interrogation.

\subsubsection{Contexte négatif}

La négation adverbiale par pas constitue le contexte le plus étudié, il n'y a pas de doute que ce type de contexte donne lieu à l'alternance qui nous préoccupe ici (12). Les négations réalisées avec les autres seminégations — au sens de Muller (1991) : personne, rien, jamais, plus... — semblent également capables de donner naissance à l'alternance (13).

(12) Max ne croit pas que Claude $\left\{\right.$ est $_{\mathrm{IND}} /$ soit $\left._{\mathrm{SBJ}}\right\}$ malhonnête.

(13) a. Personne ne croit que Claude $\left\{\mathrm{est}_{\mathrm{IND}} / \mathrm{soit}_{\mathrm{SBJ}}\right\}$ malhonnête.

b. $\quad$ Rien ne permet de penser que Claude $\left\{\right.$ est $\left._{\mathrm{IND}} / \mathrm{soit}_{\mathrm{SBJ}}\right\}$ malhonnête.

c. Une mère n'a jamais l'impression que son enfant $\left\{\operatorname{est}_{\mathrm{IND}} /\right.$ soit $\left._{\mathrm{SBJ}}\right\}$ malhonnête.

d. Marc ne pense plus que Claude $\left\{\right.$ est $_{\mathrm{IND}} /$ soit $\left._{\mathrm{SB}}\right\}$ méchant.

L'exemple (13b) permet de mentionner un autre aspect important de ces contextes : il n'est pas nécessaire que la négation s'applique sur le verbe qui enchâsse le plus directement la complétive : on rencontre des cas où il suffit d'une négation dans la matrice pour légitimer une alternance dans une complétive bien plus profonde dans la structure (14). Une étude détaillée des propriétés de ces contextes reste à faire à notre connaissance, sans doute en relation avec les contextes de légitimation du ne dit explétif.

(14) a. Max pense que Claude $\left\{\operatorname{est}_{\mathrm{IND}} /\right.$ soit $\left._{\mathrm{SBJ}}\right\}$ malhonnête.

b. Max ne pense pas que Claude $\left\{\right.$ est $_{\mathrm{IND}} /$ soit $\left._{\mathrm{SBJ}}\right\}$ malhonnête.

c. Cette histoire me fait penser que Claude $\left\{\right.$ est $_{\mathrm{IND}} /$ soit $\left._{\mathrm{SBJ}}\right\}$ malhonnête.

d. Cette histoire ne me fait pas penser que Claude $\left\{\right.$ est $\left._{\mathrm{IND}} / \mathrm{soit}_{\mathrm{SBJ}}\right\}$ malhonnête.

e. Tout le monde dit que cette histoire me fait penser que Claude $\left\{\right.$ est $_{\mathrm{IND}} / *^{*}$ soit $\left._{\mathrm{SB}}\right\}$ malhonnête.

f. Personne ne dit que cette histoire me $\left\{f^{a i t_{\mathrm{IND}}} / \mathrm{fasse}_{\mathrm{SBJ}}\right\}$ penser que Claude $\left\{\operatorname{est}_{\mathrm{IND}} / \mathrm{soit}_{\mathrm{SBJ}}\right\}$ malhonnête.

On doit enfin observer que beaucoup de prédicats concernés ont la propriété de se prêter à une interprétation «à montée de la négation » (neg-raising), selon laquelle la forme (15a) s'interprète sémantiquement comme (15b).

(15) a. Max ne trouve pas qu'il faille investir d'argent.

b. = Max trouve qu'il ne faut pas investir d'argent.

Cette observation a conduit beaucoup d'auteurs à rapprocher neg-raising et condition d'alternance. Même si ce rapprochement ouvre des pistes intéressantes, il faut noter d'une part que le neg-raising s'observe avec 
desw prédicats qui ne donnent pas lieu à l'alternance indicatif/subjonctif, par exemple le verbe vouloir qui demande le subjonctif dans tous les cas (16); et d'autre part que tous les prédicats d'opinion qui nous intéressent ici ne font pas l'objet d'un neg-raising. Ainsi, (17a) n'est pas préférentiellement interprété comme (17b).

(16) a. Paul ne veut pas que Jean s'en $\left\{* \mathrm{va}_{\mathrm{IND}} / \mathrm{aille}_{\mathrm{SBJ}}\right\}$.

b. = Paul veut que Jean ne s'en aille pas.

(17) a. Il n'est pas certain que Jean $\left\{\right.$ ment $_{\mathrm{IND}} /$ mente $\left._{\mathrm{SBJ}}\right\}$.

b. $\neq$ Il est certain que Jean ne ment pas.

Nous revenons brièvement sur ce phénomène de neg-raising un peu plus loin.

\subsubsection{Contexte interrogatif}

Selon Huot (1986), le subjonctif n'est pas permis lorsqu'il s'agit d'une interrogation marquée par la seule intonation (18b). Togeby (1966) va dans le même sens, puisque, selon lui, seule l'interrogation avec inversion du clitique sujet favorise réellement le subjonctif. Selon Huot, le subjonctif est aussi exclu dans les interrogations en est-ce que (18c). Dans l'inversion complexe (18d), l'emploi du subjonctif paraît douteux. En somme, les deux contextes les plus favorables au subjonctif seraient le contexte interrogatif avec inversion clitique (18a) et le contexte interro-négatif sans inversion clitique (18e). Si on peut suivre ces auteurs sur le caractère préférentiel de ces constructions, l'exclusion des autres formes interrogatives semble trop radical, et il est relativement facile de trouver des données qui remettent en cause cette généralisation : par exemple, la phrase (18f) est une déclarative questionnante avec l'emploi du subjonctif et elle ne nous semble pas déviante, alors que sa variante (18g) est problématique pour tous nos informateurs. De même, la phrase (18h), qui contient une question enchâssée, nous semble acceptable. Une étude plus poussée reste clairement à faire, pour mettre au jour les paramètres pertinents.

a. Crois-tu que Marie soit venue?

b. *Le directeur croit que Jean soit un bon joueur de tennis?

c. *Est-ce que le directeur croit que Jean soit un bon joueur de tennis?

(Huot, 1986)

d. ?Le directeur croit-il que Jean soit un bon joueur de tennis?

e. Tu ne crois pas que Jean soit un bon candidat?

f. On est sûr que ce soit lui au moins?

(France Inter, 13/03/2013)

g. *Tu es sûr que ce soit lui ?

h. Ça ne me dit pas quand on est sûr que ce soit Lui avec qui on aimerait finir sa vie ?

(forum forum . aufeminin. com, mars 2014)

\subsubsection{Distribution}

Il n'est pas vraiment établi quel mode est le plus fréquent dans les contextes NON-AFF, et notamment dans le contexte négatif. Les grammaires traditionnelles, plus ou moins prescriptives, présentent le mode subjonctif comme le mode le plus normal. À l'inverse, l'étude sociolinguistique de Poplack (1990, p. 22) sur le français oral parlé dans la région d'Ottawa conclut que l'indicatif est favorisé dans ce contexte : «les verbes d'opinion au négatif, qui cumulent avec les hésitations de l'histoire la condition supplémentaire de ne plus entraîner le subjonctif que lorsque la proposition principale est non-affirmative, défavorisent fortement l'emploi du subjonctif. » Une autre étude de corpus, réalisée par Sand (1983) à partir de corpus présentant le français oral parlé dans les régions de Caen et d'Orléans, mène à une conclusion assez proche 
de celle de Poplack : «Après ces verbes, employés dans des propositions négatives, on trouve le subjonctif aussi bien que l'indicatif. La tendance à l'indicatif semble être la plus forte ». L'étude de Vandergheynst (1982) aboutit à la même conclusion, bien que ses données soient peu nombreuses. Ce contexte serait un contexte qui favorise les deux modes dans la langue parlée, avec peut-être une préférence pour l'indicatif. Mais les études restent, à notre connaissance, très peu nombreuses, et aucune ne décrit précisément les préférences et les facteurs pesant dans le choix du mode dans ces contextes. Les recherches en corpus, même écrit, montrent en tout cas un comportement pas toujours systématique comme en témoignent les données suivantes, issues de deux questionnaires en ligne.

(19) a. Si vous jouez ou avez joué aux MMO, pensez vous qu'il y $\mathrm{a}_{\mathrm{IND}}$ une forme de discrimination positive envers les femmes? (Questionnaire en ligne : « Le sexisme dans les jeux vidéos »)

b. Si vous jouez ou avez joué aux MMO, pensez vous qu'il y $\mathrm{a}_{\mathrm{IND}}$ une forme d'exclusion des femmes?

c. Pensez vous que le fait d'être une femme est $\mathrm{IND}_{\mathrm{IND}}$ un atout ou un inconvénient dans le milieu du jeu vidéo?

(Ibid.)

d. Pensez vous que l'homosexualité ait ${ }_{\text {SBJ }}$ sa place dans le monde du jeu vidéo ?

(Ibid.)

a. Pensez-vous que la force de la chaîne France 3 soit $_{\text {SBJ }}$ sa dimension régionale?

(Questionnaire en ligne : «Votre perception de France 3 m'intéresse »)

b. Pensez-vous que la chaîne met IND $_{\text {D }}$ en place des moyens pour améliorer son image et pour la rendre plus jeune et attractive?

(Ibid.)

\subsubsection{Synthèse}

Le phénomène qui nous intéresse est donc caractérisé par deux éléments qui ont été notés par tous les observateurs :

- La légitimation du mode subjonctif dans les complétives de certains prédicat épistémiques par la présence d'une négation portant directement ou indirectement sur le prédicat;

— La « résistance » du mode indicatif, qui reste utilisé dans ces contextes, ce qui donne donc lieu à une alternance

Si tous les chercheurs sont d'accords sur l'existence de ce phénomène, ainsi que sur la liste des prédicats concernés, de nombreuses questions n'ont pas été tranchées, ou prêtent encore à débat. Ainsi, l'influence de la forme de négation ou d'interrogation sur la disponibilité du mode subjonctif est une question encore ouverte, qui nécessiterait que de nouvelles données soient considérées, qu'elles soient obtenues par des méthodes expérimentales ou par des analyses de corpus (voir section 4.1.2, pour quelques considérations sur Frantext, qui se révèle insuffisant pour ce type de question, à cause du manque de données). De même, de façon cruciale, la distribution effective des deux modes n'est pas établie : on va de l'idée que le mode subjonctif est le mode "obligatoire" dans les contextes NON-AFF (l'indicatif étant alors marginal, voire erroné), à des descriptions qui donnent la primauté à l'indicatif (au moins dans certains registres), en passant par l'idée que l'alternance est vraiment aléatoire, ce qui devrait conduire à une répartition identique des deux modes (toutes choses égales par ailleurs). Notre ambition a été de contribuer à répondre à cette dernière question, en établissant avec des données d'origine expérimentale la distribution des deux modes, au moins dans certains contextes. C'est ce résultat que nous présentons à la section 3. Avant cela, nous évoquons dans la section qui vient quelques-unes des explications qui sont proposées dans la littérature. 


\subsection{Explications}

Les théories sémantiques qui s'intéressent au subjonctif prédisent souvent l'emploi du subjonctif dans les contextes NON-AFF. Il y a donc un accord général sur ce premier point. En revanche, elles se distinguent sur la question du statut de l'indicatif dans ces contextes (autorisé, marginal, interdit...) et surtout sur l'éventuelle différence de sens entre les deux modes, et son explication.

\subsubsection{Rôle sémantique du subjonctif et prédiction de l'alternance}

On trouve dans la littérature diverses analyses qui considèrent que le mode subjonctif est le mode normal, attendu - sur le plan sémantique - dans les contextes NON-AFF : le contenu propositionnel n'est pas pris en charge par le locuteur (dans certains cas par le sujet, ou par un énonciateur). Ce refus de prise en charge peut correspondre soit à un engagement négatif du locuteur sur la vérité du contenu de la subordonnée, soit à un défaut d'engagement, à une position d'incertitude. Or il arrive souvent que négativité et incertitude soit décrites, dans les contextes épistémiques, comme étant du ressort du subjonctif. Ainsi, voici ce qu'écrit Confais (2002) : « l'IND vaut pour un domaine allant de $\langle$ certain $\rangle$ à $\langle$ presque certain $\rangle$, le SUBJ commence dès le $\langle$ fort possible $\rangle$ pour aller jusqu'à $\langle$ exclu / faux $\rangle$, c'est-à-dire qu'il couvre tout l'éventail du $\langle$ non certain $\rangle$ ». Cette vision est confortée par le comportement des verbes négatifs comme douter, qui appellent normalement le subjonctif (21a), mais qui, sous la négation, prenant une valeur assertive (ne pas douter que $p \approx$ affirmer que $p$ ) se retrouvent à accepter l'indicatif (21b). On aurait donc un mécanisme systématique qui concernerait aussi bien le mode indicatif que le mode subjonctif.

a. Paul doute que nous $\left\{{ }^{*}\right.$ pouvons $_{\mathrm{IND}} /$ puissions $\left._{\mathrm{SBJ}}\right\}$ réussir cette fois.

(Godard et al., 2013)

b. Paul ne doute pas que nous $\left\{\right.$ pouvons $_{\mathrm{IND}} /$ puissions $\left._{\mathrm{SBJ}}\right\}$ réussir cette fois.

Cette vision souffre d'un défaut bien connu, qui est que le subjonctif est aussi employé dans des contextes où la prise en charge par le locuteur ne fait guère de doute. Voir pour illustration les exemples (22) parmi de nombreux autres.

a. Mais il y a en vérité autre chose chez Jeanne qui explique qu'elle soit ${ }_{\mathrm{SBJ}}$ pucelle.

b. Dommage que tous mes invités ne puissent ${ }_{\mathrm{SBJ}}$ pas venir.

Par ailleurs, la prédiction de cette vision serait que le subjonctif est le seul mode disponible dans ces contextes NON-AFF : l'alternance n'est pas prédite.

D'un autre côté, certains linguistes justifient l'emploi de l'indicatif dans les contextes NON-AFF en avançant que c'est le mode qui est lexicalement associé au prédicat recteur lui-même. Ainsi, pour Moignet et al. (1981), l'alternance s'explique par le fait que deux éléments divergents peuvent être pris en compte : soit les circonstances du discours, et on a le mode qui correspond sémantiquement à tout le contexte phrastique, à savoir le subjonctif, soit la qualité sémantique du seul verbe recteur, et on a le mode habituellement associé à ce verbe recteur, à savoir l'indicatif. Grevisse \& Goosse (2008) expliquent aussi l'alternance par un phénomène lexical : c'est la présence lexicale du prédicat recteur qui appelle le mode indicatif. On aurait ainsi une association lexicale entre le prédicat et le mode indicatif. Godard (2012) synthétise ces analyses en proposant de voir l'indicatif comme un mode en cours de grammaticalisation : son association avec certains prédicats n'a pas de valeur sémantique, le subjonctif restant motivé sémantiquement dans les contextes NON-AFF. La conséquence de ces analyses est bien souvent l'absence de considération d'une quelconque différence sémantique entre indicatif et subjonctif dans les contextes NON-AFF. Enfin, pour Confais (2002), 
l'emploi de l'indicatif est analogique : on aurait une analogie entre les contextes NON-AFF et les contextes CAN - sur la base de l'identité du prédicat recteur évidemment - les premiers empruntant le mode des seconds. Toutes ces différentes analyses ressortissent à la même approche, qui voit dans l'indicatif un mode pas forcément justifié sur le plan sémantique et donc, un mode pas forcément prédictible à cet endroit par les théories (sémantiques s'entend). C'est pourquoi l'emploi de l'indicatif est souvent perçu comme illogique, ce que rappelle Confais (2002, p. 330), qui met en avant une autre logique : « la loi du moindre effort (en termes plus nobles : la force de l'analogie) parvient çà et là à opérer quelques brèches dans le système, au grand dam des puristes, par exemple : l'emploi du SUBJ après ne pas douter [...], l'emploi de l'IND dans la langue parlée après les verbes d'opinion employés négativement ». L'emploi de l'indicatif est illogique si l'on se place à un niveau purement sémantique; à un niveau lexical et dans une perspective diachronique, il est tout à fait « logique ». Toujours est-il qu'il apparaît comme une «brèche dans le système », que l'on peut choisir d'accepter comme telle ou non. Ainsi beaucoup d'analyses raccordent tout de même l'emploi de l'indicatif dans les contextes épistémiques NON-AFF au système sémantique des modes.

On aurait donc deux visions possibles :

- soit le subjonctif est motivé sémantiquement, ce qui ne prédit pas la présence de l'indicatif dans ces contextes, et qui postule une valeur sémantique spécifique au subjonctif, ce qui est clairement remis en question par la présence du subjonctif dans d'autres contextes;

- soit l'alternance résulte d'une tension entre mode lexical et mode contextuel, sans que cela corresponde à une différence de sens sémantique.

Il nous semble qu'il existe une troisième voie entre ces propositions résumées un peu brutalement : cette troisième voie est inspirée par l'impression très largement partagée que dans les cas d'alternance les deux modes ont un « effet » interprétatif différent. La question cruciale est celle de l'origine de cet effet. Nous allons proposer ici une origine pragmatique à cet effet, qui serait le résultat d'une prise en considération de la compétition entre les deux modes également légitimes sur le plan syntaxico-sémantique.

Pour élaborer cette proposition, il convient de considérer avec attention les différences que l'on peut trouver entre les contextes qui prennent l'indicatif et ceux qui prennent le subjonctif.

\subsection{2 Éléments pour une différentiation}

2.2.2.1. Items à polarité négative

Les contextes qui nous intéressent, faisant intervenir une négation, sont propices à l'apparition d'items à polarité négative, comme la préposition de à valeur indéfinie ou temporelle, la conjonction $n i$, la locution lever le petit doigt, ou les expressions quoi que ce soit, le moindre N, grand-chose, etc. (Fauconnier, 1975) :

a. *Paul croit que Jean a la moindre solution.

b. Paul ne croit pas que Jean ait la moindre solution.

Mais il est intéressant de noter que l'alternance ne semble plus si libre lorsqu'on est en présence d'items à polarité négative :

(24) Je ne crois pas qu'il y $\left\{* \mathrm{a}_{\mathrm{IND}} / \mathrm{ait}_{\mathrm{SBJ}}\right\}$ de différences entre ces deux reproductions.

(25) Je ne pense pas que Fifi $\left\{*_{\mathrm{I}_{\mathrm{IND}}} / \mathrm{ait}_{\mathrm{SBJ}}\right\}$ dormi de la nuit. [adapté de (Prince, 1976)]

(26) a. Je ne crois pas que des déclarations précises à ce sujet soient ${ }_{\mathrm{SBJ}}$ opportunes ni souhaitables.

(P. Mendès-France, Euvres complètes. 3. Gouverner, c'est choisir. 1954-1955.,1986 ; Frantext) 
b. ?Je ne crois pas que des déclarations précises à ce sujet sont ${ }_{\mathrm{IND}}$ opportunes ni souhaitables.

Les différents items ne sont pas tous aussi contraignants, comme le montre (27a), suggéré par un relecteur ; on trouve aussi des situations intermédiaires (27b).

a. Le commissaire ne croit pas que le témoin $\left\{\mathrm{a}_{\mathrm{IND}} / \mathrm{ait}_{\mathrm{SBJ}}\right\}$ vu quoi que ce soit d'intéressant.

b. Le chef ne pense pas que $\operatorname{Max}\left\{?_{\mathrm{a}_{\mathrm{IND}}} / \mathrm{ait}_{\mathrm{SBJ}}\right\}$ levé le petit doigt pour nous aider.

Descriptivement, il semble qu'on puisse considérer, comme le fait Prince (1976, p. 412), que lorsqu'il y a une montée de la négation, le subjonctif est requis, la présence d'items à polarité négative dans la complétive étant prise ici comme la trace de cette montée de la négation. Est-ce qu'on pourrait aller jusqu'à poser que l'indicatif est incompatible avec la montée de la négation ? C'est ce qu'avance Gaatone (2003), en opposant « insularité » de la proposition à l'indicatif et «non-insularité » de la proposition au subjonctif :

Dans la $1^{\text {re }}$ phrase [Je ne pense pas qu'il dort], il s'agit, dans la subordonnée d'un discours rapporté, qui se trouve hors de la portée de la négation, laquelle aurait orienté le verbe d'opinion penser vers le doute, et rendu le subjonctif possible, sinon obligatoire. Dans la $2^{\mathrm{e}}$ [Je ne pense pas qu'il dorme], la subordonnée est incluse dans la portée de la négation et le subjonctif devient possible, et sans doute préférable. En d'autres termes, il n'y a pas eu « montée de la négation » dans la $1^{\text {re }}$ ou, si l'on préfère une autre terminologie, il y a « insularité », c'est-à-dire indépendance sémantique par rapport à la principale [...], contrairement à la non-insularité de la $2^{\mathrm{e}}$, marquée par le subjonctif.

Cette question de la co-occurrence des items polaires avec l'indicatif mériterait des études plus approfondies, en lien avec le phénomène de neg-raising qui pose une difficulté spécifique dans la mesure où la nuance entre interprétation neg-raising et interprétation in situ est systématiquement délicate à établir pour les prédicats d'opinion (28), contrairement à ce qui se passe avec d'autres verbes à neg-raising comme vouloir (29).

a. L'entraîneur n'estime pas que Jean soit prêt.

b. =? L'entraîneur estime que Jean n'est pas prêt.

c. $=$ ? Ce n'est pas le cas que l'entraîneur estime que Jean est prêt.

[neg-raising] [in situ]

a. Le directeur ne veut pas que Jean parte.

b. = Le directeur veut que Jean ne parte pas.

c. $\neq$ Le directeur n'a pas la volonté que Jean parte. [neg-raising] [in situ]

Nous retenons que l'indicatif bloque les items à polarité négative, ce que nous prenons comme le signe qu'il empêche la montée de la négation (ce qui ne veut pas dire, et Prince (1976) insistait déjà sur ce point à juste titre, que le subjonctif implique une montée de la négation).

\subsubsection{Négation métalinguistique vs. négation descriptive}

On observe une différence entre emploi de l'indicatif et du subjonctif, qui découle de l'usage de la négation dans la matrice. Ainsi, quand la négation est métalinguistique, l'indicatif semble plus naturel et préférable au subjonctif :

(30) a. Je ne crois pas que Pierre $\left\{\mathrm{est}_{\mathrm{IND}} / *^{*} \mathrm{soit}_{\mathrm{SBJ}}\right\}$ grand, mais (qu'il est) immense.

b. Je ne crois pas que Pierre $\left\{\right.$ est $_{\mathrm{IND}} /$ soit $\left._{\mathrm{SBJ}}\right\}$ souple, mais (qu'il est) sportif. 
Le discours (30a) présente quelques-unes des caractéristiques de la négation métalinguistique, en particulier le fait qu'une lecture littérale de la négation conduit à une contradiction, et le subjonctif ne semble pas accessible, ce que montre le contraste avec (30b) qui ne donne pas lieu à une lecture métalinguistique de la négation.

Nous retenons que lorsque l'interprétation est nettement orientée vers une lecture métalinguistique de la négation, l'indicatif semble préféré. Ceci est compatible avec le constat fait en 2.2.2.1, puisqu'en cas de lecture métalinguistique, il n'y a pas de neg-raising.

On en déduit que dans les contextes qui nous intéressent, le subjonctif fonctionne comme une forme non marquée, par contraste avec l'indicatif qui, lui, semble compatible avec un emploi marqué, et une lecture échoïque caractéristique de la négation métalinguistique. Comme précédemment, il convient de noter que notre généralisation descriptive n'établit de contrainte que dans un sens : une négation métalinguistique implique le mode indicatif (ce qui revient à dire que le mode subjonctif empêche la lecture métalinguistique), mais le mode indicatif n'implique pas nécessairement une lecture métalinguistique de la négation.

\subsubsection{Présupposition factive}

On a pu avancer que la différence entre l'indicatif et le subjonctif était corrélée à une différence de présupposition. Ainsi, après certains verbes de perception semi-factifs comme remarquer, on observe une différence :

a. Sophie n'a pas remarqué que l'actrice $\mathrm{a}_{\mathrm{IND}}$ fait ce geste sur scène.

b. Sophie n'a pas remarqué que l'actrice ait ${ }_{S B J}$ fait ce geste sur scène.

Le verbe remarquer, lorsqu'il est suivi de l'indicatif, est factif : (31a) présuppose que l'actrice a fait «ce » geste sur scène. Au contraire, quand le subjonctif est employé (31b), la présupposition est perdue : (31b) ne présuppose pas que l'actrice a fait « ce » geste.

Schlenker (2005) propose d'étendre cette analyse au cas du verbe nier, qui n'est normalement pas factif, mais qui accepte une alternance indicatif/subjonctif (indépendamment de la polarité de la matrice), comme on le voit en (32a). Il propose que les deux variantes se distinguent par la présence (avec l'indicatif) ou l'absence (avec le subjonctif) d'une présupposition factive, ce qui expliquerait le contraste entre (32a) et (32b) : dans ce dernier cas, l'inacceptabilité de l'indicatif viendrait de l'incompatibilité de la présupposition avec le contenu asserté (le locuteur ne peut pas à la fois présupposer une proposition et la nier).

a. Jean nie qu'il \{ pleut $_{\mathrm{IND}} /$ pleuve $\left._{\mathrm{SBJ}}\right\}$

(Schlenker, 2005)

b. Je nie qu'il \{\# pleut $_{\mathrm{IND}} /$ pleuve $\left._{\mathrm{SBJ}}\right\}$

Sans endosser le principe quelquefois formulé qu'il y a une correspondance entre le mode indicatif et la présence d'une présupposition factive, on retiendra que le mode indicatif semble être celui qui est compatible avec la présupposition factive.

\subsubsection{Synthèse}

Voici comment nous proposons de synthétiser les observations de cette section à propos de la différentiation entre mode indicatif et mode subjonctif, avec les prédicats qui nous concernent : 
1. Dans la plupart des cas et dans les cas canoniques (un individu exprime une croyance sur une possible proposition du monde réel), il n'y a pas de différence fondamentale entre la phrase à l'indicatif et la phrase au subjonctif.

2. Cependant, on observe des contextes propices à l'emploi de l'indicatif, ou, pour le dire autrement, il semble que dans certaines situations marquées (pas de neg-raising, négation métalinguistique, présence contextuelle d'une présupposition) l'indicatif soit l'objet d'une préférence nette, le subjonctif étant réservé aux cas non marqués, sans doute les plus fréquents.

Nous n'avons pas d'explication à proposer sur le fait que le mode indicatif joue le rôle de mode marqué, nous soupçonnons cependant qu'une étude en diachronie pourrait éclairer cet aspect.

3. Cette capacité de l'indicatif conduit fréquemment à l'idée selon laquelle l'indicatif est plus apte à transmettre l'idée que le contenu propositionnel est assumé par un autre individu que le sujet de la croyance négative. Nous proposons d'analyser cet « effet » interprétatif comme le résultat d'un calcul essentiellement pragmatique : c'est le choix d'une forme marquée (l'indicatif), dans un contexte d'alternance, qui donne lieu aux effets interprétatifs évoqués dans cette section, et non pas une valeur sémantique qui serait intrinsèquement associée au mode indicatif.

Notons avant de clore cette section que cette position que nous prenons n'implique pas qu'il soit impossible de prédire les emplois indicatifs dans les contextes NON-AFF : comme pour tous les phénomènes pragmatiques, on s'attend à une certaine sensibilité au contexte, ce qui est compatible avec l'aproche multifactorielle que nous proposons à la fin de cet article.

Auparavant, nous présentons les résultats de l'expérience que nous avons réalisée, qui visait à nous fournir des données robustes sur l'alternance.

\section{Expérience de complétion}

Cette expérience vise à évaluer la productivité des modes subjonctifs et indicatifs dans le contexte des verbes épistémiques niés, par comparaison avec d'autre verbes et d'autres contextes. Notons que cette expérience se rapproche par son objet de l'expérience rapportée par Godard et al. (2013), qui a été réalisée à peu près en même temps que la nôtre, mais de façon indépendante, et avec un autre protocole (jugements d'acceptabilité). Les résultats semblent converger largement.

\subsection{Protocole}

\subsubsection{Tâche}

On présente aux sujets des phrases à compléter en faisant appel à leur imagination, de manière à former une phrase naturelle. La portion à compléter contient une matrice dont le verbe principal attend une complétive, le sujet de la complétive étant fourni lui aussi, ainsi que le verbe à utiliser, qui est présenté à l'infinitif. Les items se présentent donc ainsi :

Les deux sœurs ne sont pas contentes que leur père

(comprendre) 
Pour éviter que les sujets se sentent engagés dans une tâche de conjugaison, on a veillé à la disposition graphique du verbe requis qui apparaît au dessus du champ à remplir.

\subsubsection{Conditions}

Deux paramètres sont manipulés dans cette expérience : d'une part le type du prédicat, et d'autre part la polarité de la matrice. On distingue les prédicats canoniquement suivis de l'indicatif $\left(\mathrm{V}_{\mathrm{IND}}\right)$ et ceux qui sont canoniquement suivis du subjonctif $\left(\mathrm{V}_{\mathrm{SBJ}}\right)$. On a choisi :

- Des prédicats épistémiques $\left(\mathrm{V}_{\mathrm{IND}}\right)$ : croire, penser, (être) certain, avoir l'impression

- Des prédicats factifs émotifs $\left(\mathrm{V}_{\mathrm{SBJ}}\right)$ : (être) content, (être) surpris, s'étonner, regretter

La combinaison de ces deux paramètres conduit à quatre conditions possibles. Pour réduire le nombre de conditions, nous avons exclu la combinaison $\mathrm{V}_{\mathrm{SBJ}}+$ matrice niée, la condition $\mathrm{V}_{\mathrm{SBJ}}+$ positif étant suffisante pour établir la productivité du subjonctif, l'objectif principal étant de comparer les conditions $\mathrm{V}_{\mathrm{IND}}+$ positif/négatif. On a donc trois types de phrase possibles différents :

- $\mathrm{V}_{\mathrm{SBJ}}+$ positif (POS_Vs) : Les deux sœurs sont contentes

que leur père ....... (comprendre) $\ldots . .$. .

- $V_{\text {IND }}+$ positif (POS_Vi) : Les deux sœurs sont certaines

que leur père ....... (comprendre) $\ldots . .$. ..

- $\mathrm{V}_{\mathrm{SBJ}}+$ négatif (NEG_Vi) : Les deux sœurs ne sont pas certaines que leur père

(comprendre)

\subsubsection{Contraintes (sur les items)}

C'est une caractéristique générale de ce type de tâche de complétion qu'il faut porter une attention particulière au choix des items pour tenter de contraindre les productions induites à exhiber les caractéristiques attendues dans l'expérience. Dans le cas présent, il s'agissait de garantir d'une part que l'on était dans un contexte d'alternance, autrement dit que le locuteur était réellement devant un choix, d'autre part que les données produites étaient exploitables.

1. Temps verbal de la phrase matrice. La phrase matrice est au présent de l'indicatif. Ainsi, on a plus de chances de voir apparaître un tiroir présent ou passé composé dans la complétive, ce qui détermine les conditions de l'alternance.

2. Sujet de la matrice. Le sujet est toujours à la troisième personne (P3), soit au singulier, soit au pluriel, afin de neutraliser le facteur de la personne.

3. Sujet de la subordonnée. Là encore, on met une P3. On choisit des descriptions définies ou des expressions quantifiées (comme tout le monde).

4. Verbes à conjuguer. Sur le plan morphologique, on doit évidemment proposer aux sujets des verbes qui vont pouvoir faire l'objet d'une distinction formelle entre indicatif et subjonctif. Cette distinction doit être phonétique et non pas seulement orthographique : en effet, on doit prévoir le fait que les sujets ne maîtrisent pas forcément l'orthographe, qui de toute façon, apparaît comme une dimension secondaire. On fait l'hypothèse que les sujets n'utiliseront pas l'imparfait et le plus-que-parfait du subjonctif. On doit donc trouver des formes verbales permettant une distinction de mode au présent ainsi qu'au passé composé. Or, de nombreux verbes présentent des formes homophones à la P3 et/ou à la P6. C'est d'ailleurs ce qui nous a conduit à abandonner l'idée de laisser les sujets choisir leur verbe : le risque était trop grand d'avoir une forme synchrétique ambiguë. Nous choisissons donc des verbes qui permettent la distinction. Il faut ainsi éviter ces verbes (ou bien la personne à laquelle l'ambiguité est présente) : 
— Les verbes en -ER : P3 et P6 identiques : parle(nt $)_{\mathrm{IND}} /$ parle $(n t)_{\mathrm{SBJ}}$.

— Les verbes en -IR : P6 identique : finissent $_{\mathrm{IND}} /$ finissent $_{\mathrm{SBJ}}$.

- Les verbes en -RE : P6 identique : prennent $_{\mathrm{IND}} /$ prennent $_{\mathrm{SBJ}}$.

- Verbes en -OIR : P3 et P6 identiques : voit $_{\mathrm{IND}} / \mathrm{voie}_{\mathrm{SBJ}}$, doivent $_{\mathrm{IND}} /$ doivent $_{\mathrm{SBJ}}$.

Les verbes irréguliers et très fréquents distinguent en général très bien les deux paradigmes (aller, être, faire, savoir). Nous les avons utilisés en priorité. Mais nous avons aussi choisi des verbes dont la forme de subjonctif présent se différencie plus minimalement de la forme à l'indicatif (connaître, prendre, mettre, dire, devoir). Ils sont proposés pour la P3 car il y a souvent homophonie à la P6.

Sur le plan syntaxique, les verbes choisis ne sont pas des verbes intransitifs et sous-catégorisent au moins un complément, afin que les sujets puissent être le plus possible dans une activité créatrice. Ils peuvent aussi entrer dans des constructions à verbe support : être, faire, prendre, mettre, dire. Certains peuvent encore être auxiliaires ou semi-auxiliaires, comme être, aller et devoir.

Enfin, les verbes ont souvent un sens assez général, afin, là encore, de mobiliser la dimension créatrice.

\subsubsection{Déroulement}

Le nombre d'items produits a été calculé pour que chaque sujet voie 8 fois chaque condition, ce qui fait 24 phrases en tout, et l'expérience comportait autant de distracteurs. L'expérience a été programmée sur Ibex Farm, et proposée sous la forme d'un questionnaire en ligne. Quelques sujets ont été sollicités directement par nos soins parmi les étudiants présents à l'université, mais l'expérience a aussi été déposée sur le site du RISC, Expesciences, et c'est par ce biais que sont venus la plupart des sujets. Nous avons recueilli 120 questionnaires, dont 9 ont été éliminés pour des raisons techniques.

Les données présentées exploitent donc 111 questionnaires. Un sujet a été éliminé (le français n'était pas sa langue maternelle), et les phrases produites ont été manuellement codées pour le mode choisi par les sujets.

\subsection{Résultats}

\subsubsection{Attentes}

Le résultat mesuré était donc le choix de l'indicatif ou du subjonctif dans la phrase produite. Les résultats attendus peuvent être résumés ainsi :

- Dans la condition $\mathrm{V}_{\mathrm{SBJ}}+$ positif (POS_Vs), on s'attend à trouver toujours le subjonctif : la présence de choix de l'indicatif devrait être interprétée comme une non accessibilité du subjonctif pour le sujet (ce qui à son tour peut être interprété comme une absence dans l'idiolecte du sujet, ou bien comme la considération que le subjonctif n'était pas pertinent dans le registre en question).

— Dans la condition $\mathrm{V}_{\mathrm{IND}}+$ positif (POS_Vi), on s'attend à trouver toujours l'indicatif : la présence d'un subjonctif serait interprétée comme une sur-correction, ou un problème de compétence lexicale.

- La condition $\mathrm{V}_{\mathrm{IND}}+$ négatif (NEG_Vi) est la condition cible de l'expérience : selon la plupart des auteurs, on devrait rencontrer le subjonctif, et c'est ce que disent aussi en général les grammaires prescriptives. Mais la plupart des linguistes ont souligné la présence d'une alternance dans ce contexte, et on s'attend donc plutôt à une répartition des deux modes, l'un des résultats attendus étant une mesure de cette répartition, qui pourrait permettre de mettre au jour une tendance. 


\subsubsection{Mesures brutes}

La comparaison "brute" entre les modes est résumée dans le tableau et le graphique de la figure 1.

Comparaison brute IND-SUBJ

\begin{tabular}{l|r|r||r|r|r} 
& \multicolumn{2}{|c||}{ IND } & \multicolumn{2}{c|}{ SUBJ } & \multirow{2}{*}{632} \\
\cline { 2 - 5 } NEG_Vi & 155 & $24,53 \%$ & 477 & $75,47 \%$ & 632 \\
POS_Vi & 625 & $97,96 \%$ & 13 & $2,04 \%$ & 638 \\
POS_Vs & 42 & $6,58 \%$ & 596 & $93,42 \%$ & 638
\end{tabular}

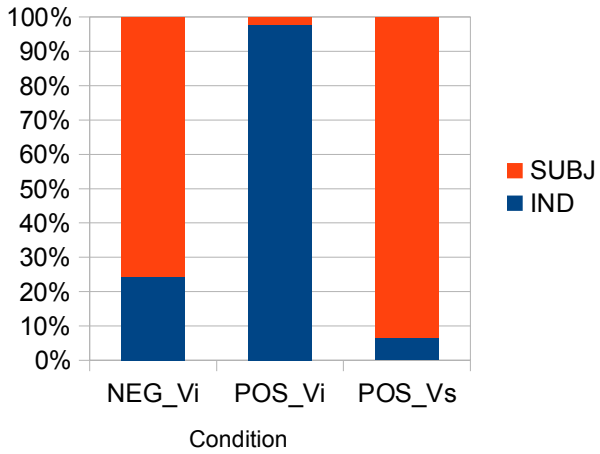

Figure 1. Mesure brute des emplois des modes indicatifs/subjonctif

Les deux colonnes de droite donnent un résultat conforme à nos attentes : les prédicats appelant l'indicatif sont produits avec l'indicatif dans $97,96 \%$ des cas, les prédicats appelant le subjonctif sont produits avec le subjonctif dans $93,42 \%$ des cas, on peut donc considérer que la productivité du subjonctif ne doit pas être remise en cause : au moins pour ces verbes, et pour ces sujets, le subjonctif fait partie des modes accessibles et relevant de leur compétence.

Dans la condition-cible de notre expérience, on constate que la tendance vers l'emploi du subjonctif est très nette, puisqu'il est produit dans plus de 75\% des cas, mais il n'en reste pas moins que dans près d'un quart des cas, c'est l'indicatif qui est choisi. Même si les pourcentages précis doivent être pris avec précaution, on peut conclure que la préférence pour le subjonctif dans un contexte négatif est très franche, mais pas absolue.

\subsubsection{Mesures corrigées}

Les résultats précédents nous renseignent sur la productivité effective des deux modes dans les conditions que nous avons créées. Mais si l'on se rappelle que les deux modes ne sont pas toujours dans une compétition " égalitaire », il convient de considérer un aspect supplémentaire : dans certains cas, le choix des temps verbaux pour la complétive peut modifier les conditions d'alternance ( $c f$. discussion plus haut sur les temps verbaux). Nous avons donc procédé à une annotation plus fine des données, pour être en mesure de distinguer les cas où le choix est réellement libre (le sujet exprime clairement une complétive au présent, et se trouve vraiment devant l'alternative indicatif/subjonctif) et les cas où les deux modes ne sont pas également disponibles (par exemple si le sujet exprime un procès futur dans la complétive, son choix peut se faire entre le futur morphologique, forcément à l'indicatif, et un présent du subjonctif qui prend alors une valeur future, mais ne commute pas avec le présent de l'indicatif (34)).

$$
\text { Paul ne pense pas que son père }\left\{\text { comprendra }_{\text {IND-FUT }} / \text { comprenne }_{\text {SBJ-PRE }} / \text { ?comprend }_{\text {IND-PRE }}\right\} \text { un jour. }
$$

Cette annotation plus fine est présentée à la figure 2, avec les mesures corrigées obtenues en se limitant aux cas les plus stricts d'alternance. Le détail des annotations est donné dans le tableau 1. 

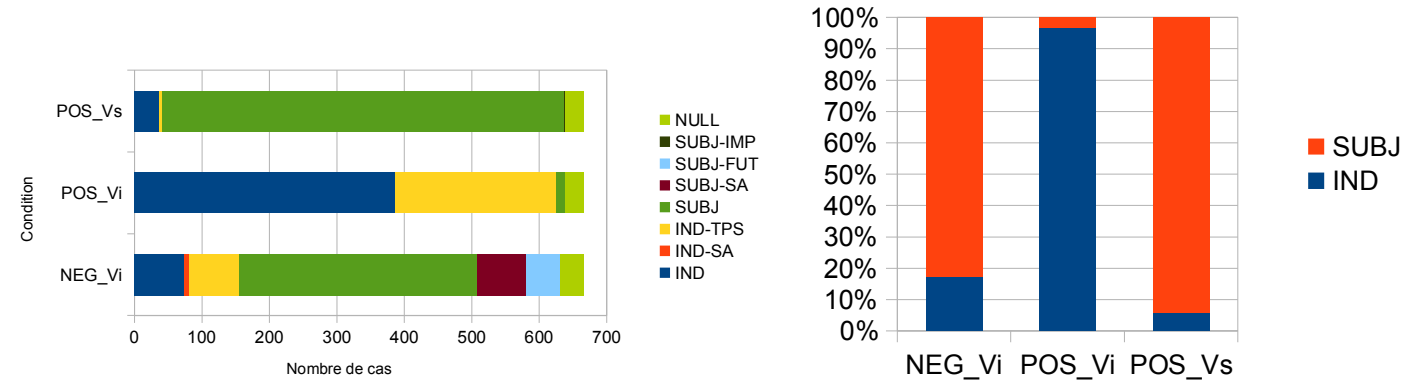

Figure 2. Distribution détaillée des modes selon les cas d'alternance, et mesure corrigée des emplois des modes indicatif/subjonctif

\begin{tabular}{|l|l|}
\hline IND & $\begin{array}{l}\text { indicatif présent ou passé composé } \\
\text { indicatif à un autre temps } \\
\text { IND-TPS } \\
\text { SUbJjonctif présent ou passé composé } \\
\text { SUBJ-FUT } \\
\text { subjonctif présent à valeur temporelle future (le subjonctif alterne alors avec les indicatifs } \\
\text { futurs). Ce codage sert à noter les subjonctifs qui s'interprètent le plus naturellement avec } \\
\text { une valeur 'futur'. } \\
\text { subjonctif imparfait, disparu en français contemporain courant, employé seulement à deux } \\
\text { reprises (et de façon fautive). } \\
\text { SUBJ-IMP }\end{array}$ \\
$\begin{array}{l}\text { la forme utilisée est le semi-auxiliaire devoir ou pouvoir alors que ce n'était pas demandé. } \\
\text { On met à part cette catégorie car il se pourrait que ce type de semi-auxiliaire influence } \\
\text { IND-SA }\end{array}$ \\
NUULL & $\begin{array}{l}\text { l'emploi du subjonctif. } \\
\text { Erreur : pas de verbe conjugué, forme ambiguë... }\end{array}$ \\
\hline
\end{tabular}

Tableau 1. Détail de l'annotation des cas d'alternance

Comme on le voit, la forme générale des résultats n'est que très peu affectée par ce raffinement : le seul changement significatif est la légère baisse de la proportion d'indicatifs produits dans la condition NEG_Vi, qui passe en dessous de $20 \%$. Il reste que l'on peut conclure, et d'une façon plus assurée que précédemment, que si le subjonctif est l'objet d'une préférence forte dans la condition qui nous intéresse, il faut expliquer que les locuteurs choisissent de produire l'indicatif dans une proportion de l'ordre d'un cas sur cinq.

\subsubsection{Discussion(s)}

Disposant d'un nombre important de données, nous avons procédé à plusieurs analyses, pour tenter de mettre en évidence l'influence de facteurs pertinents. Nous avons vérifié la significativité des différences observées par rapport aux sujets et au items. Pour le reste, notre échantillon étant assez important, nous avons tenté de mesurer l'impact de l'âge et de la catégorie socio-professionnelle comme facteur, et n'avons mis en évidence aucune tendance significative en ce qui concerne ces deux variables. Faute de place, nous ne pouvons développer ces analyses ici.

La variabilité entre les sujets est assez importante, mais il faut noter que tous les sujets produisent du subjonctif en grande quantité, au moins dans la condition de contrôle POS_Vs. 


\subsubsection{Synthèse}

Sans préjuger des résultats d'analyses supplémentaires encore en cours, on peut conclure qu'il existe, en français contemporain, une classe de prédicats qui sont le lieu d'une tension entre le mode indicatif qui leur est lexicalement associé et le mode subjonctif dans les contextes NON-AFF. Cette tension est résolue le plus souvent en faveur du subjonctif, puisque c'est le mode qui est choisi dans 75 à $80 \%$ des cas, mais toutes choses égales par ailleurs, l'indicatif reste choisi dans une proportion notable de cas, et ce fait n'a reçu jusqu'à présent aucune explication.

Nous tirons de cette synthèse deux conclusions : d'une part, il est peu probable que l'on se trouve devant un phénomène catégorique : il semble illusoire de chercher quelle règle (ou combinaison de règles) de la grammaire peut à la fois expliquer que l'association lexicale entre un prédicat et un mode doit céder le pas dans certains contextes devant l'influence du contexte, et que cette préférence ne s'applique que dans 4 cas sur 5. En revanche, et c'est notre seconde conclusion, il semble qu' une approche multi-factorielle soit assez prometteuse : elle permettrait de réconcilier une distribution des données non catégorique et le sentiment largement répandu - et confirmé par nos données — que le choix dans l'alternance n'est pas purement aléatoire.

\section{Vers une analyse multi-factorielle}

Les considérations précédentes orientent notre futur programme de recherche : nous pensons qu'il est pertinent de mettre au jour soigneusement la liste, vraisemblablement longue et hétéroclite, des facteurs qui semblent pouvoir exercer une influence sur le choix du mode dans les contextes d'alternance.

La liste qui est présentée dans cette section n'est qu'un premier pas dans cette direction, et représente les éléments que nous avons commencé d'annoter en vue d'une exploitation statistique dont nous disons quelques mots dans la section 4.2 .

\subsection{Facteurs possibles}

\subsubsection{Le sujet du verbe de la matrice : quelle personne?}

Souvent, les études évoquent le lien entre première personne (comme sujet de la matrice) et subjonctif : la première personne favoriserait plus le subjonctif que l'indicatif, ou bien la première personne favoriserait plus le subjonctif que les autres personnes. C'est par exemple l'avis de Boysen (1969). Si le subjonctif est effectivement particulièrement présent lorsque le sujet de la matrice est à la première personne, cela pourrait accréditer la thèse selon laquelle le subjonctif marque une distance du locuteur avec le contenu propositionnel. Nous avons fait une recherche succincte dans Frantext, parmi les textes de 1970 à de nos jours. Le subjonctif est majoritaire, de façon écrasante, après la première personne. Pour la troisième personne, les données sont malheureusement peu nombreuses. On constate cependant qu'indicatif et subjonctif y sont utilisés à part égale : on trouve 7 subjonctifs et 7 indicatifs, en écartant le futur de l'indicatif. Les données ne sont pas assez nombreuses pour pouvoir en tirer une généralité, mais la troisième personne a tout de même l'air d'un cas moins clair — c'est-à-dire d'un cas où il y a moins clairement un mode préféré — que la première. Pour la deuxième personne, il est encore plus rare de trouver un contexte négatif traditionnel, c'est-à-dire déclaratif (comme Tu ne crois pas que Marie est malhonnête) ; nous n'en avons pas trouvé dans Frantext. 


\subsubsection{Le type de la phrase (niveau syntaxico-pragmatique)}

Nous parlons ici du type de la phrase globale dans laquelle apparait la complétive. Nous nous sommes jusqu'à présent attardés sur le type déclaratif. Mais la négation peut être employée dans d'autres types de phrases. Il serait intéressant de voir ce qui s'y passe. Sur Frantext, l'indicatif est écrasant après les interronégatives sans inversion clitique (30 indicatifs et aucun subjonctif), qui ont en fait la forme d'une phrase déclarative négative (35). Ces dernières présentent toutes un sujet à la deuxième personne.

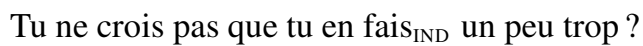

(G. Dormann, La Petite main, 1993 ; Frantext)

On comprend que l'acte de langage qui se joue ici soit particulier et explique l'emploi de l'indicatif. Il s'agit pour le locuteur de présenter une proposition de façon déclarative à son interlocuteur, afin qu'il la valide ou non. La négation ne porte pas ici sur le contenu propositionnel (il n'y a pas montée de la négation semble-t-il), mais sur le verbe épistémique uniquement. Le contexte impérativo-négatif (pour la deuxième personne toujours) est un contexte d'alternance : indicatif et subjonctif sont tous deux utilisés (on relève 12 subjonctifs et 6 indicatifs sur Frantext) :

(36) a. Ne crois pas que je sois $\mathrm{IND}_{\mathrm{ND}}$ à la recherche de distractions.

(J.-B. Pontalis, Loin, 1980 ; Frantext)

b. Ne crois pas que tu n'es ${ }_{\text {SBJ }}$ pas la bienvenue parmi nous!

(C. Aventin, Le Cœur en poche, 1988 ; Frantext)

\subsubsection{Le verbe conjugué et sa morphologie (niveau lexico-morphologique)}

On peut penser que l'emploi des modes dépend aussi du verbe conjugué lui-même, plus précisément de sa morphologie. Ainsi, on pourrait imaginer que le subjonctif est plus employé quand il s'agit de formes verbales supplétives par exemple, ces formes étant morphologiquement saillantes. En effet, les formes de subjonctif sont très différentes en fonction des verbes. Parfois les formes du subjonctif et de l'indicatif sont très proches, sans parler des formes homophones, qui sont un cas bien particulier, parfois elles sont très différentes. Pour les formes très proches, on peut citer : $\mathrm{dit}_{\mathrm{IND}} / \mathrm{dise}_{\mathrm{SBJ}}$, sort $_{\mathrm{IND}} / \mathrm{sorte}_{\mathrm{SBJ}}$. Les formes très différentes sont en général celles des verbes irréguliers et très fréquents, qui sont souvent supplétives : est $_{\mathrm{IND}} / \mathrm{soit}_{\mathrm{SBJ}} \mathrm{va}_{\mathrm{IND}} /$ aille $_{\mathrm{SBJ}}$. On pourrait envisager que les formes plutôt proches peuvent se confondre : les locuteurs emploieraient alors plus facilement la forme indicative, qui créerait, dans un contexte favorable au subjonctif, un effet de disjonction moindre. À l'inverse, les formes bien identifiables et fréquentes seraient peut-être plus facilement stockées en mémoire et employées par les locuteurs.

Ce serait ici un facteur qui ne serait pas propre au contexte que nous envisageons.

\subsubsection{Autres facteurs}

D'autres facteurs ont été évoqués au long de cet article, comme la forme de la négation ou de l'interrogation, l'influence des items lexicaux eux-mêmes (nous n'avons décelé aucune différence significative entre les $\mathrm{V}_{\mathrm{IND}}$ et les $\mathrm{V}_{\mathrm{SBJ}}$ que nous avons choisis, mais il ne faut surtout pas en déduire que tous les verbes donnent nécessairement lieu à l'alternance dans les mêmes conditions), l'influence des temps verbaux de la matrice et de la subordonnée, sans parler de facteurs plus discursifs, prenant en compte le contexte conversationnel plus large, dont l'influence a été soupçonnée par certains auteurs. 


\subsection{Méthode}

Sans pouvoir présenter de résultats à ce stade, nous voulons plaider pour une étude de la question qui nous concerne avec la méthode présentée par Bresnan et al. (2007) pour l'alternance dative en anglais. Assumant résolument l'idée que le choix indicatif/subjonctif dans les contextes d'alternance est sous l'influence d'une constellation de facteurs divers, et ne peut être analysé comme obéissant à des règles catégoriques, il s'agit, par un travail d'annotation très détaillé, de construire sur corpus un modèle prédictif au moyen de méthodes statistiques (par exemple la régression logistique) qui nous permette d'expliquer la distribution des emplois effectivement rencontrée en corpus.

Cette méthode requiert une mise au jour soigneuse de l'ensemble des facteurs pertinents, tels qu'ils ont été relevés dans la littérature, qui peuvent aller du lexique à la pragmatique, mais avec l'objectif, au lieu de choisir entre ces facteurs, comme ont été conduits à le faire certains linguistes, d'établir statistiquement leur influence relative.

\section{Synthèse}

Nous intéressant à l'alternance des modes indicatif/subjonctif en français, et en nous restreignant à un contexte spécifique (les prédicats d'opinion sous la négation), nous avions dans cet article un double objectif. D'une part présenter les résultats d'une expérience de production induite, qui mène à la conclusion que l'on a bien affaire à une alternance productive. D' autre part, proposer une première liste de facteurs qui doivent être envisagés, ensemble, pour une analyse factorielle de l'alternance, que nous préconisons (et prévoyons) d'aborder avec un travail sur corpus et l'utilisation de méthodes statistiques (régression logistique) dont la pertinence s'est manifestée dans de nombreux champs de la linguistique ces dernières années.

\section{Remerciements et déclaration de contribution}

Les auteurs souhaitent vivement remercier Barbara Hemforth, pour son aide lors de la préparation du matériel expérimental ; Emilia Ellsiepen, qui nous a aidé pour l'analyse des données ; Danièle Godard, pour de nombreuses discussions sur le subjonctif; Pierre Larrivée et un relecteur anonyme, qui nous ont aidé à clarifier certains passages de cet article. Nous remercions aussi les nombreux participants de nos expériences. Il va sans dire que les erreurs et imprécisions qui pourraient rester sont de notre seule responsabilité.

Cette recherche a bénéficié d'un soutien du programme ANR-10-LABX-0083 (Labex “Empirical Foundations of Linguistics").

Les recherches bibliographiques ont été réalisées par Floriane Guida dans le cadre de son Master (Guida, 2013); le matériel expérimental a été élaboré par Floriane Guida, avec l'aide d'Emilia Ellsiepen et Pascal Amsili; la passassion des expériences a été menée par les deux auteurs; les résultats ont été annotés par Floriane Guida, et analysés par Pascal Amsili (avec l'aide d'Emilia Ellsiepen). Les deux auteurs ont travaillé ensemble à la rédaction de l'article. 


\section{Références bibliographiques}

Boysen, G. (1969). Le mode de la proposition complétive préposée dans les langues romanes. Revue romane, 4(1), $10-19$.

Bresnan, J., Cueni, A., Nikitina, T., Baayen, R. H. et al. (2007). Predicting the dative alternation. Cognitive foundations of interpretation, p. 69-94.

Confais, J.-P. (2002). Temps, mode, aspect: les approches des morphèmes verbaux et leurs problèmes à l'exemple du français et de l'allemand. Interlangues, linguistique et didactique. Presses Universitaires du Mirail.

Fauconnier, G. (1975). Polarity and the scale principle. In Papers from the eleventh Regional Meeting of the Chicago Linguistic Society, CLS: University of Chicago.

Gaatone, D. (2003). La nature plurielle du subjonctif français. In P. Hadermann, A. van Slijcke \& M. Berré, Eds., $L a$ syntaxe raisonnée. Mélanges de linguistique générale et française offerts à Annie Boone à l'occasion de son 60ème anniversaire, p. 57-78. Louvain-la-Neuve: De Boeck-Duculot.

Godard, D. (2012). Indicative and subjunctive mood in complement clauses: from formal semantics to grammar writing. In C. Piñón, Ed., Empirical Issues in Syntax and Semantics 9, p. 129-148. Publication en ligne ISSN 1769-7158.

Godard, D., Hemforth, B. \& Marandin, J.-M. (2013). Negation and mood alternation in French. A preliminary experimental investigation of polarity mood. Manuscrit proposé en hommage à Ivan Sag. http://www . stanford. edu/ dept/linguistics/structures_evidence/.

Grevisse, M. \& Goosse, A. (2008). Le Bon Usage. Bruxelles: de Boeck-Duculot, 14e edition.

Guida, F. (2013). Deux modes pour un contexte. L'alternance des modes indicatif et subjonctif en francais contemporain. Mémoire de master 2 recherche, Université Paris Diderot.

Huot, H. (1986). Le subjonctif dans les complétives: subjectivité et modalisation. In M. Ronat \& D. Couquaux, Eds., La grammaire modulaire, p. 81-101. Paris: Éditions de Minuit.

Moignet, G., Cervoni, J., Schlyter, K. \& Vassant, A. (1981). Systématique de la langue française. Editions Klincksieck.

Muller, C. (1991). La négation en français. Genève: Droz.

Mørdrup, O. (1975). Présuppositions, implications et verbes français. Revue Romane, 10, 125-157.

Poplack, S. (1990). Prescription, intuition et usage: le subjonctif français et la variabilité inhérente. Langage et société, 54(1), 5-33.

Prince, E. F. (1976). The syntax and semantics of Neg-Raising, with evidence from French. Language, 52(2), 404-426.

Sand, J. U. (1983). Le subjonctif en français oral. In Actes du VIIIe Congrès des Romanistes Scandinaves, Odense University Classical Studies: International Specialized Book Service Incorporated.

Schlenker, P. (2005). The lazy Frenchman's approach to the subjunctive: Speculations on reference to worlds and semantic defaults in the analysis of mood. In Proceedings of Going Romance XVII.

Togeby, K. (1966). La hiérarchie des emplois du subjonctif. Langages, 3, 67-71.

Vandergheynst, P. (1982). L'emploi des modes et des temps dans les subordonnées introduites par que, observations sur un corpus de français parlé. Mémoire de licence, Katholieke Universiteit te Leuven.

Wilmet, M. (1997). Grammaire critique du Français. Hachette Supérieur/Duculot.

1. Outre l'impératif (Que l'accusé se taise SBJ $_{\mathrm{J}}$ ), il existe quelques cas d'emploi du subjonctif dans une principale (Je ne sache ${ }_{\mathrm{SB}}$ pas que le problème soit résolu.), mais nous pouvons négliger ces emplois dans le présent travail. 